\title{
¿Cómo traducir el lunfardo al alemán? \\ El reto de la variación lingüística en la traducción audiovisual ${ }^{*}$
}

\author{
Eva Gugenberger (Leipzig)
}

\begin{abstract}
This article focuses on the problems arising from non-standard varieties in the translation of audiovisual texts using the example Lunfardo. This special repertoire of words and phrases, which originated in the late 19th century in the lower classes in Buenos Aires, has become a commonly used and characteristic part of colloquial Rioplatense Spanish. Taking the example Okupas, an Argentine TV series for young people, and guided by the priority function that the use of Lunfardo has in the series, this study intends to identify an appropriate style of speech for dubbing into German. Based on a selected dialogue, some results are presented that were developed in class together with students of translation. The objective of the analysis is to demonstrate that it is possible to find more creative and adequate solutions to go further than the usual practice to level diasystematic characteristics when translating into standard German.
\end{abstract}

\section{$1 \quad$ Planteamiento del tema}

La presencia de una variedad lingüística no-estándar en un texto audiovisual supone un gran reto para el traductor o la traductora que se enfrenta a ella. Es sabido que en la práctica de la traducción, sobre todo en el doblaje, muchos traductores optan por la neutralización de la misma, transfiriéndola a un lenguaje que se aproxima al estándar de la lengua meta con tan solo algunas inserciones lexicales de índole diasistemática para marcar emblemáticamente el trasfondo sociocultural de los personajes.

Esta práctica habitual se debe a varios factores, entre otros, a las expectativas de los espectadores de la versión traducida. Estamos acostumbrados a que en películas dobladas al alemán incluso niños de la calle de México o ladrones argentinos hablan en alemán estándar, aunque así se pierde mucho de la atmósfera del contexto original. Si se traduce -por ejemplo-chumbo por 'Pistole' en vez de 'Knarre', se evoca -en términos de la semántica de scenes-andframes- otra escena en los espectadores alemanes, otra imagen del ambiente social en el que se desarrolla la historia completamente distinta a la intencionada originalmente.

\footnotetext{
* Esta es una versión revisada de un artículo publicado en: Reutner, Ursula/Rentel, Nadine/Schröpf, Ramona (eds.) (2015): Traducción audiovisual y lingüística contrastiva en los medios en diálogo con la Filología Hispánica. Frankfurt a. M. etc., Lang: 141-162.

Linguistik online 86, 7/17 - http://dx.doi.org/10.13092/lo.86.4118
}

CC by 3.0 
Otra razón por la cual se suele optar por la transferencia al estándar es que muchos especialistas en traducción están convencidos de que es sumamente difícil, o incluso imposible, encontrar un equivalente válido y apropiado en la lengua meta, sobre todo si se trata de un dialecto geográfico. Sin duda la marcación diatópica plantea el mayor problema a la hora de buscar una solución satisfactoria, ya que los dialectos están íntimamente vinculados a determinadas regiones geográficas en la conciencia de los hablantes. La elección de un dialecto determinado de la comunidad linguíística de llegada implica una disonancia con el trasfondo cultural de la película (cf. Herbst 1994: 98). Incluso puede producir un efecto cómico no pretendido en la versión original, si por ejemplo un campesino de la región andina habla en la variedad bávara en alemán.

En cuanto a la variación social se considera menos complicada su traducción, "siempre y cuando los contextos situacionales y la organización social sean relativamente equiparables en ambos polisistemas" (Mantarro 2010: 161; apoyándose en Rabadán 1991: 115). En todas las sociedades suelen existir clases y grupos sociales cuyas hablas se diferencian por rasgos linguiísticos específicos. Sin embargo, tampoco es tarea fácil encontrar estrategias de traducción apropiadas para variedades diastráticas, sobre todo porque suelen solaparse con la dimensión diatópica. Como indica Herbst (1994: 89), no existe ninguna variedad que no esté marcada social y regionalmente al mismo tiempo. Así por ejemplo, el habla de un obrero del décimo distrito de Viena muestra rasgos dialectales de esta ciudad, pero también marcas sociolectales del estrato social al que pertenece.

El traductor o la traductora tiene que tomar su decisión de acuerdo con el aspecto que considera prioritario en el texto original. Si la marcación social es más importante, puede servirse de un sociolecto correspondiente en la lengua meta. En cambio, si prevalecen los rasgos regionales para caracterizar un personaje, tratará de reproducir esta peculiaridad en el contexto de llegada, ya sea mediante un dialecto real o bien mediante la creación de un lenguaje artificial. Por esta última solución se optó en el doblaje de la película francesa Bienvenue chez les Ch’tis (Boon 2008) al alemán, que resultó un gran éxito.

Como se ha constatado muchas veces en la literatura especializada (cf. Herbst 1994; Pöllmann 2009; Mantarro 2010), el uso de la variación lingüística en un texto audiovisual nunca es casual, más bien siempre tiene una función determinada. El guionista se sirve de ella como recurso estilístico para que los diálogos parezcan más auténticos y naturales, para hacer referencia a la pertenencia de ciertos personajes a una cultura, una región o un grupo social específicos, y con la finalidad de lograr un efecto determinado, por ejemplo el efecto de identificación en los espectadores de la cultura de llegada. Por lo tanto, la pregunta clave de la que tiene que partir el traductor o la traductora es por la función que cumple la variación lingüística en el texto original y cómo se puede mantener esta función -en la medida de lo posible- en la versión traducida.

Teniendo en cuenta todo esto, las siguientes páginas tratarán del lunfardo como recurso estilístico en un texto audiovisual y del reto de transmitirlo al alemán. Después de ilustrar brevemente el desarrollo del lunfardo desde su origen hasta la actualidad y de situar este fenómeno en el diasistema del español, se abordará la función que cumplen los lunfardismos en el caso concreto de Okupas (Stagnaro 2000), una serie de televisión rodada en Argentina en el año 2000. Luego se tratará de identificar el estilo de habla más adecuado en la lengua meta, el

ISSN 1615-3014 
alemán, para el lenguaje de los protagonistas en la serie. Tomando como ejemplo un diálogo de Okupas, finalmente se presentarán las propuestas de traducción para los lunfardismos encontrados en él, fruto de la labor realizada con estudiantes en un curso de traducción en la Universidad de Hildesheim.

Nuestro objetivo en clase fue elaborar una traducción preliminar que sirva como base para el doblaje del diálogo (sin tomar en cuenta la sincronicidad de los labios). Fijamos como destinatarios de la versión alemana a adolescentes y adultos jóvenes del espacio lingüístico germanófono; como canal y horario de emisión se optó por el canal 3SAT en banda horaria de tarde.

De acuerdo con las líneas de la estrategia traductológica pragmática que ha propuesto Herbst (1994: 237-239, 250) para el doblaje, determinamos como objetivos principales:

- equivalencia de la función del texto, es decir, que se permita concebir el texto meta como si se tratase de una versión original, manteniendo las funciones principales del texto de partida.

- $\quad$ equivalencia del sentido original del texto, es decir, que los enunciados en la lengua meta sean adecuados en una situación equiparable a la de partida, teniendo en cuenta el sentido que surge del uso de una variedad no-estándar. Si bien no es posible lograr una equivalencia total de las variedades original y de meta, no queríamos traducir simplemente al alemán estándar, sino encontrar enunciados que evoquen un efecto -por lo menos similar- en los espectadores de la cultura meta.

Pero, ¿qué significa esto en el contexto concreto? Para poder contestar esta pregunta, hay que averiguar primero qué es el lunfardo y cuál es su valor en la cultura de partida, es decir, en la sociedad rioplatense; y segundo, cuál es la función de las expresiones de lunfardo que utilizan los personajes en la serie Okupas.

\section{$2 \quad$ El lunfardo}

El lunfardo surgió en la segunda mitad del siglo XIX en los arrabales de Buenos Aires, donde argentinos de las clases marginadas, "gente de mal vivir" como ladrones, compadritos, proxenetas, prostitutas, etc. vivían puerta con puerta con inmigrantes de distinto origen étnico. El grupo de inmigrantes más numeroso lo constituían los italianos, lo que explica la gran cantidad de lunfardismos derivados del italiano. Desde aquel entonces el lunfardo se suele tildar como lenguaje de los delincuentes, lo que es un concepto erróneo, ya que se comete el error de tomar una parte por el todo (cf. Teruggi 198: 26). Gobello señala al respecto:

Y puesto que en el caudal de una inmigración que no fue selectiva llegaron delincuentes por carradas, es natural que muchos términos lunfardos sean de origen delincuencial. Sin embargo, afirmar que el lunfardo es un lenguaje de los delincuentes porque cuenta con un número relativamente vasto de voces carcelarias sería más o menos como sostener que el castellano es un dialecto árabe porque se formó en la etapa de la dominación musulmana en España y porque incluye muchos términos arábigos.

(Gobello 2004)

Desde los arrabales el lunfardo se extendió rápidamente, incorporándose en las hablas coloquiales de Buenos Aires y -seguidamente- de toda la región del Río de la Plata. A su expansión contribuyó también su uso en las canciones de tango y en los sainetes criollos, un género de teatro popular que tuvo su auge en las primeras décadas del siglo XX en Argentina. A par- 
tir de los años cincuenta se inició una ampliación y dinamización del repertorio del lunfardo, lo que se debe mayormente a la emergencia de las culturas juveniles urbanas. Al crear sus estilos comunicativos ${ }^{1}$, los jóvenes fueron sirviéndose de material lingüístico de diferentes variedades y registros. Incorporaron muchas voces del lunfardo -manteniendo o cambiando el significado original de ellas- e inventaron nuevos lunfardismos, de manera que vinieron y siguen desarrollando este repertorio lingüístico de manera creativa. El nuevo lunfardo es como lo expresa Teruggi (1978: 27) - “obra de la juventud”. Por esta razón, podemos decir que hoy en día el lunfardo es característico de las hablas juveniles, aunque hay una cantidad considerable de términos que también son utilizados por otros sectores de la sociedad rioplatense.

El lunfardo no se puede clasificar de forma inequívoca en el diasistema de la lengua española. Como repertorio de lexemas y modismos, así como algunos fenómenos morfológicos ${ }^{2}$, no constituye una variedad singular distinta. Más bien se hace uso de términos lunfardos -con mayor o menor frecuencia- en las variedades no-estándares del castellano rioplatense. Koch/Österreicher (2011: 259) lo clasifican como argot localizado geográficamente, así que lo ubican dentro de la dimensión diatópica y diastrática. En la terminología de estos dos autores se puede decir que una serie de lunfardismos ha ascendido en la cadena de las variedades desde la diastrática a la diafásica, a saber, que se usa por hablantes de distinto origen social en situaciones informales.

En los diccionarios de lunfardo -como en el de Gobello y Gottero- las entradas se adscriben a diferentes niveles lingüísticos. Gobello (1990: 10) distingue entre las siguientes marcaciones:

- Lenguaje general: Esta categoría corresponde al "léxico oficial corriente"; según el diccionario de Gottero (2006), son "expresiones sumamente comunes", que no han quedado en las clases populares y bajas, sino que se usan también en otros sectores de la sociedad.

- Expresiones populares: las que se pueden utilizar también por miembros de la "buena sociedad" en sentido humorístico.

- Expresiones groseras: "es lo que ninguna persona de buen gusto dice, como no sea en rueda muy íntima".

- Expresiones características de determinados grupos sociales (delincuentes, etc.)

- Lunfardismos tradicionales (términos traídos por la inmigración)

\footnotetext{
${ }^{1}$ Al utilizar aquí el término estilo comunicativo me baso en la argumentación de Androutsopoulos (2006), quien concibe el lenguaje juvenil como estilos comunicativos sociales. Esto corresponde a un enfoque sociolingüístico que se orienta en los hablantes como actores sociales y no en el sistema lingüístico, o sea, que interpreta la variación linguiística a partir del contexto situacional y desde la perspectiva de los hablantes.

${ }^{2}$ Estos comprenden diferentes tipos de metaplasmos, es decir, alteraciones de palabras por supresión, adición o transposición. Los más frecuentes son la paragoge, que consiste en la adición de una letra o sílaba al final de la palabra (p. ej. truchardi por 'trucho', celuloide por 'celular') (cf. Plass 2013: 18) y la metátesis o permutación de sílabas, fenómeno que se conoce por vesre (p. ej. gotan por 'tango').
}

ISSN 1615-3014 
Al consultar en estos dos diccionarios las entradas de los lunfardismos detectados en el diálogo elegido de Okupas que vamos a ver más abajo, resulta que la gran mayoría de ellos corresponde a la categoría "popular":

- lenguaje general: che

- lenguaje popular: onda, al bardo, al pedo, largar algo, pegar algo, tener culo, cargar a alguien, joder a alguien, mandar a la guerra a alguien, chumbo, sanatear

- lenguaje popular y grosero: boludo, orto

- lenguaje delictivo: comerse a alguien

- lunfardo: pibe

En resumen podemos destacar lo siguiente: El lunfardo es un repertorio de palabras, modismos y algunas particularidades morfológicas, que se desenvuelve en una permanente dinámica, enriqueciendo el español rioplatense. Su uso ya no se restringe a determinados clases o grupos sociales, aunque expresiones lunfardescas aparecen con mayor frecuencia en variedades diastrática y diafásicamente marcadas como "bajas" y caracterizan de manera especial los estilos comunicativos juveniles.

\section{$3 \quad$ La función del lunfardo en Okupas}

Lo expuesto hasta ahora ya nos aporta algunos indicios valiosos, pero aún no nos ofrece una respuesta satisfactoria a la pregunta de cuáles serían las soluciones traductológicas adecuadas en un texto concreto. Por lo tanto, el siguiente paso consiste en averiguar cuál es la pertenencia sociocultural que se quiere marcar mediante un lenguaje salpicado de lunfardismos como recurso estilístico en un texto literario o audiovisual determinado. Su uso puede indicar que los personajes pertenecen al ambiente de los estafadores como en Nueve Reinas (Bielinsky 2000), una película argentina policial del año $2000 ;{ }^{4}$ o puede llevar al lector al mundo del boxeo como en el cuento Torito de Julio Cortázar (1956) (en Final del juego); o también puede situar la historia en una cultura juvenil callejera como en la serie Okupas en la que nos centramos en el presente trabajo. Dependiendo del contexto específico, posiblemente se requerirán soluciones de traducción distintas.

Entonces hay que preguntarse, cuál es la finalidad que persigue Bruno Stagnaro, el autor y director de Okupas, cuál es el efecto que quiere provocar en los espectadores al poner en boca de sus protagonistas:

- ¿qué onda? en vez de ¿qué tal?

- ¿largaste la facu? en lugar de ¿dejaste la universidad?

- ¿ ¿cómo la pegaste esta casa? en lugar de ¿cómo conseguiste esta casa?

\footnotetext{
${ }^{3}$ Otra distinción se podría hacer entre lunfardismos que se utilizan exclusivamente en la región del Río de la Plata y por ende conllevan una fuerte marca diatópica (como che, boludo, cargar a alguien en el sentido de 'tomar a alguien para la joda', 'tomar a alguien el pelo') y aquellos que -si bien fueron incorporados en los diccionarios mencionados- se registran también en otras regiones hispanohablantes (como joder a alguien o ¿qué onda?). Pero al no tener relevancia para nuestros propósitos, esta distinción no tiene que ser tratada en detalle aquí.

${ }^{4}$ Cabe mencionar aquí el excelente trabajo de Plass acerca del doblaje de Nueve Reinas al alemán, en el que propone una traducción de la película al dialecto y sociolecto de las clases bajas de Viena (Plass 2013).
} 
- ¿ ¿no me estás jodiendo? o ¿no me estás cargando? en vez de ¿no me estás tomando el pelo?

Debido a las limitaciones de espacio propias del presente trabajo y la imposibilidad de realizar un análisis detallado de la totalidad del texto original, señalaré a continuación solo los aspectos más relevantes para la traducción.

Como ya se mencionó, Okupas es una mini-serie de televisión transmitida por Canal 7 en el año 2000, durante una época en la que Argentina sufría una profunda crisis económica. Esta llevó a cineastas como Stagnaro a retratar a los jóvenes de una nueva generación sin trabajo y sin perspectivas. Okupas cuenta la historia de Ricardo (Rodrigo De La Serna), un estudiante de Medicina de clase media, y de tres eventuales amigos de él que provienen de sectores sociales bajos: El Pollo (Diego Alonso Gómez), Walter (Ariel Staltari) y El Chiqui (Franco Tirri). Juntos habitan u "ocupan" una casa, que pertenece a la prima de Ricardo, para impedir que la casa sea ocupada ilegalmente por personas sin hogar. Stagnaro no busca ni heroizar ni victimizar a sus protagonistas, más bien adopta la mirada de Ricardo que se sumerge en el mundo de la marginalidad sin mucho que perder, descubre la cultura del ladrón, el porro, la cocaína y las armas, pero también llega a conocer la lealtad entre amigos. Así los televidentes tienen un guía con el que comparten ingenuidades y, a través de sus equivocaciones y aciertos, aprenden un poco más de ese mundo tan lejano y cercano a la vez (cf. Latinpedia; s. v. Series de televisión: Okupas).

La intención prioritaria de Stagnaro no es retratar la vida de los ocupantes ilegales, que actúan por su miseria económica, ni es concientizar al público de los problemas de la gente desamparada. Más bien le interesa, como él mismo afirma, "que la historia de los cuatro pibes sea creíble" (Isola s. a.). La historia debe sugerir autenticidad, queriendo lograr un efecto de identificación en los espectadores jóvenes cuya situación y cuyos deseos son similares a los de los cuatro chicos en Okupas.

La credibilidad y la autencidad deben ser transmitidas también a través del estilo de habla de los protagonistas, "un lenguaje de la calle", que varía un poco según el trasfondo sociocultural de cada personaje. Las imágenes que deben evocar las expresiones verbales son aquellas que se vinculan con una cultura callejera juvenil. Como hemos visto, los lunfardismos forman parte del estilo comunicativo juvenil. Por supuesto, también contribuyen al colorido local de la película, por medio de ellos queda claro que los hablantes son argentinos. Pero esta marcación diatópica puede pasar a un segundo plano en el texto traducido, puesto que la intención primordial de los personajes no es demostrar su pertenencia a la cultura rioplatense, sino más bien su afiliación a un grupo social determinado. Los lunfardismos contribuyen a construir la identidad social y lingüística de una cultura urbana juvenil, cuya existencia no constituye un rasgo típicamente argentino, sino un fenómeno global, aunque se pueden encontrar peculiaridades según cada región. Los temas que preocupan a los muchachos en Okupas son relevantes en gran parte para la juventud en otros países, también en Alemania. Asimismo, el registro linguiístico de los jóvenes en todo el mundo muestra analogías, por ejemplo la tendencia a utilizar interjecciones, expresiones vulgares y determinaciones específicas para chica, chico, amigo, compañero o actividades que giran en torno al amor y el sexo, al dinero, a la droga, etc. (cf. Zimmermann 2004: 43). 
En base a estas informaciones se puede concluir que una traducción válida, que cumpla con los objetivos formulados anteriormente (equivalencia de la función y del sentido del texto, del nivel estilístico y del efecto a provocar), se nutrirá de los registros juveniles de la cultura alemana. Siguiendo el enfoque orientado en el hablante, la pregunta central resulta ser: ¿Cómo se expresarían jóvenes alemanes en una situación análoga? La tarea consiste entonces en transferir las expresiones verbales de los protagonistas de Okupas a enunciados situacionalmente adecuados, sirviéndose de un estilo comunicativo que corresponde a la cultura juvenil. Si bien es cierto que, al aplicar esta estrategia, no se transmite el carácter argentino del lenguaje de la película, se puede renunciar a transferir los rasgos diatópicos, ya que funcionalmente prevalece el carácter de "lenguaje callejero juvenil" mediante el cual los personajes manifiestan su pertenencia social y su resistencia a la cultura normativa.

\section{$4 \quad$ La traducción del lunfardo en un diálogo de Okupas}

A continuación se presenta el diálogo a traducir ${ }^{5}$, tomado del segundo capítulo de la serie titulado Bienvenidos al tren. Corresponde a una escena en la casa de la prima de Ricardo, en la cual Ricardo invita al Pollo a compartir la casa con él para cuidarla. Después de hablar sobre lo que pasa actualmente en la vida de cada uno (respecto al apartamento en $E l D_{0} k^{6}$, que El Pollo comparte con otros chicos, y respecto a los estudios de Ricardo), la conversación gira en torno a la casa. A Ricardo le cuesta un poco convencer al Pollo de la seriedad de su propuesta. Al final, pronuncia oficialmente una invitación a vivir juntos en dicha casa.

\section{Diálogo entre Ricardo y EI Pollo ${ }^{7}$ (04:33-06:03)}

$\mathrm{R}-$ ¿Qué? ¿Vivís con ella?

P - No, estoy viviendo con unos pibes en el Dock.

$\mathrm{R}$ - Y, ¿qué onda?

P - Un bardo ... un bardo. ¿Y vos? ¿Qué?

$\mathrm{R}$ - ¿Qué de qué?

$\mathrm{P}$ - ¿En qué andás?

R - ¡Qué sé yo! Al pedo estoy.

$\mathrm{P}$ - ¿Qué, largaste la facu?

R - No, la facultad me la comí.

$\mathrm{P}$ - Che, ¿y esto? ¿Cómo la pegaste esta casa?

\footnotetext{
5 Agradezco sinceramente a Kerstin Plass, sin cuya colaboración este trabajo no se hubiera podido realizar. Ella "descubrió" Okupas, puso a mi disposición la transcripción del diálogo, llevada a cabo por ella y presentada en el coloquio Sprachkontakt und Hybridität in Lateinamerika ('Contacto de lenguas e hibrididad en América Latina') en la Universidad de Viena, y me apoyó con valiosas referencias. Asimismo, mis agradecimientos van dirigidos a los estudiantes del curso Übersetzen gesprochener Sprache und Dialoge ('Traducción de lenguaje oral y diálogos'), impartido en el semestre de verano de 2012 en la Universidad de Hildesheim. Los resultados presentados aquí son fruto de su labor creativa.

${ }^{6}$ El Dock: Se refiere a Dock Sud, un distrito de clase obrera en la periferia de Buenos Aires, donde la tasa de criminalidad ha aumentado enormemente en los últimos años.

${ }^{7}$ Los lunfardismos están marcados en negrita.
} 
R - ¡Viste, boludo! Mi prima ...

P - ¡San Culo está contigo, hermano! Yo no lo puedo creer. Siempre tenés esa familia, boludo, vos ... salvadora, vos.

$\mathrm{R}$ - ¿Qué familia salvadora? ¡Si me mandó a la guerra, la hija de puta! ... ya fue ... igual me ... qué sé yo. Me viene bien estar acá. ¿Me entendés?

P - Sí, más vale.

$\mathrm{R}-i \mathrm{O}$ no?

P - Pero mirá que no ... que no es para nada fácil, loco, mantener una casa así como esta. Más, solo.

$\mathrm{R}$ - Pero bueno, ¿para qué te llamé a vos?

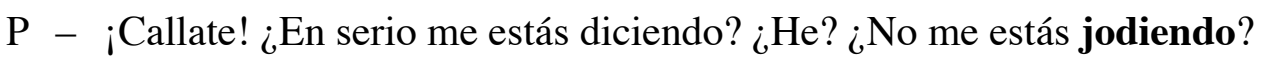

$\mathrm{R}$ - Yo pongo la casa, vos ponés el chumbo.

P - Boludo, ¿no me estarás cargando? Mirá que yo vine del Dock y no quiero volver más, boludo.

R - ¿Qué querés? ¿Que te ponga un pasacalles, que me tatúe una nalga? ¿Qué querés que haga, boludo?

P - Boludo, ¡que no me sanateés, nada más! ¡Que no me digas que es así y después me sacás a palazos!

R - ¡No te estoy sanateando! Te estoy diciendo, ¡mirá! ... En este sencillo acto ... te invito, amigo mío, a que compartas la conquista de este caserón del orto conmigo.

A manera de ejemplo, quiero resaltar y comentar el siguiente extracto:

$\mathrm{P}$ - Che, ¿y esto? ¿Cómo la pegaste esta casa?

R - ¡Viste, boludo! Mi prima ...

P - ¡San Culo está contigo, hermano!

En estos tres turnos encontramos tres marcadores discursivos típicos de Argentina, viste, che y boludo, de los cuales los dos últimos corresponden al lunfardo. No es por casualidad que se denomine a los argentinos che boludos en tono humorístico. Che, correspondiente al vocativo del pronombre $t u ́$, tiene valor de interjección, en el presente contexto es una señal de contacto para dirigirse a alguien. Como equivalente con la misma función discursiva en alemán los estudiantes propusieron 'he', 'Alter' o 'Mann'.

Boludo significa 'imbécil', 'tonto', pero tanto aquí como en la mayoría de los casos hoy en día se utiliza de manera desemantizada como interjección secundaria. Es una forma de tratamiento muy usual entre los jóvenes argentinos, que funcionalmente equivale a formas como 'Mann', 'Alter' en alemán.

La muletilla viste constituyó un obstáculo considerable en la traducción. En general, se emplea dentro o al final de un enunciado como señal de control de contacto o para lograr la afirmación del interlocutor, parecido a sabes, no, verdad, entiendes (cf. Martín Zorra- 
quino/Portolés Lázaro 1999: 4181). Pero en el presente contexto está en posición inicial y tiene más bien un valor evaluativo, reproducido muy bien en la expresión alemana 'Krass, ne?' que marca claramente la pertenencia a la cultura juvenil. Otras soluciones propuestas por los estudiantes para la exclamación entera ;Viste, boludo! son las siguientes: 'Zieh's dir rein, Alter!' 'Tja, Alter, da kannste [kannst du] mal sehen!' y 'Siehste, du Sack?'

La expresión de lunfardo pegar(se) algo corresponde a 'conseguir algo', 'obtener algo' en el estándar español. Por tanto, las soluciones traductológicas que consideramos más acertadas para el enunciado ¿Cómo la pegaste esta casa? son: 'Wie biste [bist du] an die Bude [an das Haus] (hier) rangekommen?' o 'Wie hast (du) dir denn die Hütte untern Nagel gerissen?'

Es interesante que algunos estudiantes votasen por 'Hütte' o 'Bude' en lugar de 'Haus', sosteniendo que estos lexemas marcados diastráticamente se integran mejor en el estilo general del discurso y además se adaptan bien a la imagen. De esta manera aplicaron una estrategia compensatoria, es decir, compensaron la falta de equivalentes estilísticos en otros casos, como en el siguiente turno, en el que se consideró imposible encontrar una solución totalmente satisfactoria para la expresión San Culo.

San Culo está contigo es una innovación creada por El Pollo, haciendo alusión a un santo de la suerte (que no existe). Como tener culo corresponde a 'tener suerte' en estándar, los estudiantes se decidieron por 'Schwein haben', un modismo que suaviza ligeramente la marca vulgar de la expresión de partida. Veamos las opciones que se propusieron para todo el enunciado ;San Culo está contigo, hermano!:

- 'Hast du ein Schwein, Bruder!'

- 'Junge, du hast echt Schwein gehabt!'

- 'Scheiße, du hast immer Glück, Bruder!'

- 'Alter Schwede, hast du ein Schwein [Glück]!'

- 'Na du bist ein Glückspilz!'

La forma de tratamiento hermano se tradujo por 'Bruder' o 'Junge' por la mayoría de los estudiantes. Referente a Bruder se discutió si el término no está demasiado ligado a la cultura hip-hop, proveniente del inglés brother o brow, sin llegar a un punto de vista unánime. Los que optaron por Bruder en la traducción sostuvieron que esta palabra se suele usar también como forma de tratamiento entre jóvenes independientemente del subgrupo al que pertenecían.

Cabe mencionar la solución creativa de una estudiante al proponer 'Alter Schwede' por hermano, incluyendo una permutación de los constituyentes. Este modismo coloquial se utiliza en alemán en el sentido de 'Kumpel' o sin referirse a una persona determinada como expresión que indica asombro o sorpresa.

A continuación, se presenta el diálogo en su conjunto, tanto el texto de partida como de llegada, de acuerdo con las soluciones de traducción elaboradas en clase. De la variedad de propuestas se ha elegido una y -en algunos casos- se añade alternativas en corchetes. Debajo de cada turno se pone los lunfardismos con su definición, interpretación y posibles equivalentes en alemán. En algunos casos veremos que el significado con el que se utiliza un término en la actualidad -en general y también en el texto- difiere del original. Es interesante que la mayoría de estas voces, que demuestran la evolución dentro del lunfardo, pertenezcan al lenguaje 
juvenil, a saber: ¿qué onda?, bardo, estar al pedo, pegar algo y comerse algo. En esta lista también se puede incluir boludo, porque -a pesar de no ser de uso exclusivo de la juventudse utiliza con mayor frecuencia entre los jóvenes como forma de tratamiento.

\begin{tabular}{|c|c|}
\hline Texto original & Propuestas de traducción \\
\hline $\mathrm{R}$ - ¿Qué? ¿Vivís con ella? & 'Und? Wohnst du mit ihr zusammen?' \\
\hline $\begin{array}{l}\mathrm{P}-\text { No, estoy viviendo con unos } \\
\text { pibes en el Dock. }\end{array}$ & 'Nein, ich wohn' mit ein paar Jungs im Dock.' \\
\hline pibe & $\begin{array}{l}\text { 'niño' - 'Junge', 'Bursche'. } \\
\text { Del ital. pivetto; se aplica afectivamente a personas de } \\
\text { cualquier edad }(\mathrm{GOB})^{8} \text {. }\end{array}$ \\
\hline $\mathrm{R}-\mathrm{Y}$, ¿qué onda? & 'Und, was geht da ab?' \\
\hline onda & $\begin{array}{l}\text { 'asunto', 'cuestión'. } \\
\text { Término tomado de la radiofonía ('onda electromagnéti- } \\
\text { ca: movimiento vibratorio del éter') (GOB); se usa a } \\
\text { partir de los años } 1960 \text { (CONDE)9'. } \\
\text { ¿qué onda? ¿qué tal? - 'Wie geht's?', 'Wie läuft's?' } \\
\text { buena onda - 'cool'; > persona: 'gute Person' - (col.) } \\
\text { 'cooler Typ'; > lugar: En ese bar hay buena onda - } \\
\text { (col.) 'Das ist ein cooler Schuppen'. } \\
\text { Leng. de la drogadicción: en la expresión tirar ondas } \\
\text { (buenas, malas, etc.): 'ejercer influjo' (GOB). }\end{array}$ \\
\hline
\end{tabular}

\begin{tabular}{|l|l|}
\hline $\begin{array}{l}\text { P - Un bardo ... un bardo. ¿Y } \\
\text { vos? ¿Qué? }\end{array}$ & 'Katastrophe ... totales Chaos. Und bei dir? Was?' \\
\hline bardo & $\begin{array}{l}\text { al bardo: 'improvisadamente', 'sin plan previo'; 'inú- } \\
\text { tilmente'; 'descuidadamente' - 'planlos', 'achtlos'. } \\
\text { De la expresión ladronil trabajo de albarde: 'robo en el } \\
\text { cual el ladrón va sin rumbo, sin conocimiento previo de } \\
\text { lo que va a robar'; del portugués albardar 'hacer algo de } \\
\text { prisa y descuidadamente' (GOB). } \\
\text { bardo: a partir de los años } 1980 \text { también: 'lío', 'proble- } \\
\text { ma', 'embrollo' (Wiktionary; s. v. lunfardo bardo) - } \\
\text { 'Durcheinander', 'Chaos'. }\end{array}$ \\
\hline
\end{tabular}

\footnotetext{
${ }^{8} \mathrm{GOB}=$ Gobello (1990).

${ }^{9} \mathrm{CONDE}=$ Información personal de Oscar Conde.
} 


\begin{tabular}{|l|l|}
\hline $\mathrm{R}-$ ¿Qué de qué? & 'Wie was?' \\
\hline $\mathrm{P}-$ ¿En qué andás? & 'Was gibt's bei dir so?' \\
\hline $\mathrm{R}-$ ¿Qué sé yo! Al pedo estoy. & $\begin{array}{l}\text { 'Keine Ahnung. Ich gammel' [lunger'] so rum. [Ich } \\
\text { kratz' mir nur den Arsch.]' }\end{array}$ \\
\hline pedo & $\begin{array}{l}\text { 'embriaguez' (GOB), 'borrachera'. } \\
\text { Etimología incierta (CON) }\end{array}$ \\
\hline $\begin{array}{l}\text { en pedo: 'ebrio' (GOB) - 'betrunken'. } \\
\text { al pedo: 'inútilmente' (GOB; CON) - 'unnötigerweise', } \\
\text { 'umsonst'. } \\
\text { Actualmente también: } \text { estar al pedo: 'estar sin hacer } \\
\text { nada' - 'nichts tun', (col.) 'herumhängen'. }\end{array}$ \\
\hline largar & $\begin{array}{l}\text { 'Hast(e) die Uni g'schmiss(e)n?' } \\
\text { 'entregar', 'abandonar'; 'despedir', 'echar', 'apartar de' } \\
\text { (GOB); se aplica a personas o actividades - 'aufgeben'; } \\
\text { 'loslassen', 'gehen lassen'; (col.) 'schmeißen'. } \\
\text { Del esp. largar: 'soltar', 'dejar libre' (CON). }\end{array}$ \\
\hline
\end{tabular}

\begin{tabular}{|l|l|}
\hline $\mathrm{R}$ - No, la facultad me la comí. & 'Ne, die hab' ich durchgezogen [durchgedrückt].' \\
\hline comerse & $\begin{array}{l}\text { comerse a alguien: (leng. del.) 'matar a alguien' (GOB) } \\
\text { - ‘jmd. töten'. } \\
\text { Hoy también: 'llevar a alguien a la cama' (CONDE) - } \\
\text { 'jmd. ins Bett bringen', (col.) 'abschleppen'. } \\
\text { comerse la cana: 'tragarse una detención' (PER) }{ }^{12} . \\
\text { Con un significado similar al último hoy se utiliza mu- } \\
\text { cho entre jóvenes: comerse algo: 'soportar', 'aguantar' } \\
\text { (y 'terminar') algo (CON) - 'aushalten', 'durchhalten', } \\
\text { (col.) 'etwas durchziehen'. }\end{array}$ \\
\hline
\end{tabular}

\begin{tabular}{|l|l|}
\hline $\begin{array}{l}\text { P - Che, ¿y esto? ¿Cómo la pe- } \\
\text { gaste esta casa? }\end{array}$ & $\begin{array}{l}\text { 'He, und das (Mann)? Wie hast(e) dir die Hütte untern } \\
\text { Nagel gerissen?' }\end{array}$ \\
\hline che & $\begin{array}{l}\text { Corresponde al vocativo del pronombre tú (GOB); pue- } \\
\text { de tener valor de: }\end{array}$ \\
\hline
\end{tabular}

\footnotetext{
${ }^{10} \mathrm{CON}=$ Conde (2004).

11 Apócope de facultad.

12 PER = Pérsico (2004).
} 


\begin{tabular}{|c|c|}
\hline & $\begin{array}{l}\text { 1. Interjección para dirigirse a alguien - 'he'; } \\
\text { 2. Partícula expletiva: p. ej. Y bueno, che, viste, es lo que } \\
\text { hay } \\
\text { Del español antiguo ce, voz con que se llama a una per- } \\
\text { sona (CON). }{ }^{13}\end{array}$ \\
\hline pegar & $\begin{array}{l}\text { 'gustar', 'interesar'; 'acertar'; 'lograr un éxito inespera- } \\
\text { do en algún asunto o empresa', 'triunfar'; 'comprar dro- } \\
\text { ga' (CON). } \\
\text { Del esp. pegar: 'tener efecto una cosa' (CON). } \\
\text { pegar(se): 'conseguir', 'obtener' (CON) - 'bekommen', } \\
\text { 'kommen zu'. } \\
\text { Del esp. pegar: 'unir o juntar una cosa con otra' (CON). }\end{array}$ \\
\hline
\end{tabular}

\begin{tabular}{|l|l|}
\hline $\mathrm{R}-i$ Viste, boludo! Mi prima ... & 'Krass, ne? [Zieh's dir rein, Alter!] Meine Cousine ...' \\
\hline boludo & 'imbécil', 'tonto' (GOB) - 'Dummkopf', 'Trottel', \\
& 'Idiot'. \\
& Fórmula de tratamiento, utilizada muy frecuentemente \\
& entre jóvenes - 'Mann', 'Alter', 'Kumpel'. \\
& Del esp. bolas: 'testículos', con claro sentido peyorativo \\
& $(\mathrm{CON})$. \\
\hline
\end{tabular}

\begin{tabular}{|c|l|}
\hline $\begin{array}{c}\text { P - ¡San Culo está contigo, } \\
\text { hermano! Yo no lo puedo } \\
\text { creer. Siempre tenés esa fa- } \\
\text { milia, boludo, vos ... salva- } \\
\text { dora, vos. }\end{array}$ & $\begin{array}{l}\text { 'Mensch [Junge], hast du ein Schwein! Ich fass' es } \\
\text { nicht. Du immer mit deiner rettenden Familie, Alter. } \\
\text { [Jedes Mal rettet dir deine Familie den Arsch.]' }\end{array}$ \\
\hline San Culo & $\begin{array}{l}\text { tener culo: tener suerte - 'Schwein haben'. } \\
\text { San Culo es una personificación que hace alusión a un } \\
\text { santo imaginado. } \\
\text { San Culo está contigo - 'der Heilige des Glücks ist auf } \\
\text { deiner Seite'. }\end{array}$ \\
\hline
\end{tabular}

$\mathrm{R}$ - ¿Qué familia salvadora? ¡Si 'Von wegen (rettende Familie)! Diese Scheißfamilie hat me mandó a la guerra, la hija de puta! ... ya fue ... mich ja da erst reingeritten! [Meine verfickte Familie hat Igual me ... qué sé yo. Me viene bien estar acá. ¿Me entendés? mir doch den ganzen Scheiß hier eingebrockt!] Aber was soll's! ... Egal ... (was weiß ich). Tut mir schon (ganz) gut, hier zu sein. Weißt du, was ich mein'?'

\footnotetext{
${ }^{13}$ Esta explicación etimológica, recogida por Corominas en su diccionario etimológico, es la que suele adoptarse por los lexicógrafos. Sin embargo, hay otras hipótesis como la de Pascual Asensi, quien designa el vocativo árabe yā como posible étimo de la interjección che (Pascual Asensi 2007).
} 


\begin{tabular}{|c|c|}
\hline mandar a la guerra a alguien & $\begin{array}{l}\text { guerra: 'estrategias y trucos para triunfar en una con- } \\
\text { quista amorosa, del mismo modo que se emplean las } \\
\text { tácticas para ganar una batalla' (GOTT) }{ }^{14} \text {; en alusión al } \\
\text { maquillaje dela mujer que se pinta 'como los indios } \\
\text { cuando quieren guerra” (CON) - 'Eroberungstaktik'. } \\
\text { Hoy también: 'pelea', 'camorra', 'problema'; alguien } \\
\text { que busca guerra es alguien que se busca problemas (por } \\
\text { lo general innecesarios) (Wiktionary; s. v. lunfardo gue- } \\
\text { rra) - 'Streit', 'Schlägerei', 'Problem'. } \\
\text { mandar a la guerra a alguien: 'meterle a alguien en } \\
\text { problemas' - 'jmd. in Schwierigkeiten bringen'. }\end{array}$ \\
\hline ya fue & $\begin{array}{l}\text { 'ya pasó', 'ya terminó'; a partir de los años } 1990 \\
\text { (CONDE) - '(ist) schon geschehen', '(ist) schon vorbei' }\end{array}$ \\
\hline P - Sí, más vale. & 'Na klar. [Ja, auf jeden (Fall).]' \\
\hline $\mathrm{R}-\succsim \mathrm{O}$ no? & 'Oder nicht?' \\
\hline $\begin{array}{l}\text { P - Pero mirá que no ... que no } \\
\text { es para nada fácil, loco, } \\
\text { mantener una casa así como } \\
\text { esta. Más, solo. }\end{array}$ & $\begin{array}{l}\text { 'Na ja, aber ... das ist gar nicht so einfach, so ein Haus } \\
\text { instand zu halten, Alter, schon gar nicht allein.' }\end{array}$ \\
\hline loco & $\begin{array}{l}\text { En el lunfardo tiene un significado parecido a boludo. } \\
\text { Fórmula de tratamiento entre jóvenes; se usa desde fina- } \\
\text { les de la década de } 1960 \text { (CON) - 'Mann', 'Alter'. } \\
\text { Del esp. loco: 'de poco juicio, disparatado e imprudente' } \\
\text { (CON). }\end{array}$ \\
\hline $\begin{array}{l}\mathrm{R} \text { - Pero bueno, ¿para qué te } \\
\text { llamé a vos? }\end{array}$ & $\begin{array}{l}\text { 'Na und, was meinst du, wofür ich dich wohl angerufen } \\
\text { habe? [wofür du hier bist?]' }\end{array}$ \\
\hline $\begin{array}{l}\mathrm{P}-\text { ¡Callate! ¿En serio me estás } \\
\text { diciendo? ¿He? ¿No me es- } \\
\text { tás jodiendo? }\end{array}$ & $\begin{array}{l}\text { 'Hör auf! Im Ernst? [Ohne Quatsch?] He, du verarschst } \\
\text { mich doch nicht, oder?' }\end{array}$ \\
\hline joder a alguien & $\begin{array}{l}\text { 'perjudicar'; 'fastidiar', 'molestar'; 'utilizar a una perso- } \\
\text { na como objeto de burla' - 'belästigen', 'ärgern'. } \\
\text { Del arcaísmo esp. hoder: 'practicar el coito' (GOB; } \\
\text { GOTT). } \\
\text { A partir de los años } 1960 \text { también: 'bromear', 'divertir- }\end{array}$ \\
\hline
\end{tabular}

${ }^{14}$ GOTT $=$ Gottero (2006). 


\begin{tabular}{|l|l|}
\hline se' $(\mathrm{CON})$ - 'spaßen', 'scherzen'. \\
hablar en joda: ‘hablar en broma' - 'etwas im Scherz \\
sagen'. \\
joder a alguien ( cargar a alguien $)$ - 'sich über jmd. \\
lustig machen'.
\end{tabular}

\begin{tabular}{|l|l|}
\hline $\begin{array}{l}\mathrm{R} \text { - Yo pongo la casa, vos ponés } \\
\text { el chumbo. }\end{array}$ & 'Ich stell das Haus, du die Knarre.' \\
\hline chumbo & $\begin{array}{c}\text { 'proyectil de arma de fuego'; 'revólver' - 'Kugel', 'Pis- } \\
\text { tole', (col.) 'Knarre'. } \\
\text { Del portugués chumbo: 'plomo' (GOB). }\end{array}$ \\
\hline
\end{tabular}

$\mathrm{P}$ - Boludo, ¿no me estarás cargando? Mirá que yo vine del Dock y no quiero volver más, boludo.

cargar a alguien
'Mensch, und du verarschst mich wirklich nicht? He, du weißt doch, dass ich vom Dock komm und da will ich nicht mehr hin, Mann.'

'burlarse de alguien con insistencia y desenfado'. joder a alguien - 'jmd. zum Besten halten', 'sich über jmd. lustig machen'.

Del esp. cargar: 'incomodar'; (lenguaje delictivo) 'llevar dinero con uno mismo'; (pop. y gros.) 'tener un varón grandes órganos genitales' (GOB).

$\mathrm{R}-$ ¿Qué querés? ¿Que te ponga un pasacalles, que me tatúe una nalga? ¿Qué querés que haga, boludo?

'Was willst du denn noch? Soll ich dir einen roten Teppich ausrollen? Oder soll ich es mir auf die Arschbacke tätowieren? Was willst du, dass ich tu, Mann?'
$\mathrm{P}$ - Boludo, ¡que no me sanateés, nada más! ¡Que no me digas que es así y después me sacás a palazos! sanatear
'(Alter), dass du nicht rumlaberst, ganz einfach! Dass du mir nicht sagst 'komm!' und mich dann wieder hochkant rauswirfst!'

'hablar sin fundamento' - 'herumreden'.

De sanata: 'tautología, repetición inútil de un mismo pensamiento expresado de diversas maneras' (GOB); 'discurso monótono que enuncia varias veces la misma idea, pero expresada de diferentes maneras, a fin de ocultar la carencia de una argumentación más completa o profunda' (GOTT).

Del ital. zannata: 'bufonada' (GOB); 'burla o humorada típica de los payasos (zanni)’ (GOTT). 


\begin{tabular}{|c|c|}
\hline $\begin{array}{l}\text { R - ¡No te estoy sanateando! } \\
\text { Te estoy diciendo, ¡mirá! ... } \\
\text { En este sencillo acto ... te } \\
\text { invito, amigo mío, a que } \\
\text { compartas la conquista de } \\
\text { este caserón del orto conmi- } \\
\text { go. }\end{array}$ & $\begin{array}{l}\text { 'Ne, ich mein's ernst! Also: Hiermit lade ich dich ein, } \\
\text { mein lieber Freund, diese beschissene Bruchbude mit } \\
\text { mir zu erobern.' }\end{array}$ \\
\hline orto & $\begin{array}{l}\text { 'orificio anal', 'culo', 'parte posterior o asentaderas', } \\
\text { 'trasero', 'nalgas en general'(GOB; GOTT). } \\
\text { Etimología incierta; posiblemente del esp. hortelano } \\
\text { (GOB). }{ }^{15} \\
\text { del orto } \sim \text { de mierda - 'Scheiß-', 'beschissen'. }\end{array}$ \\
\hline
\end{tabular}

Tabla 1: Diálogo entre Ricardo y El Pollo con propuestas de traducción al alemán (Okupas: Bienvenidos al tren; 04:33-06:03)

\section{$5 \quad$ Conclusión}

En este trabajo se ha abordado el problema que plantea la presencia de variedades noestándares en textos audiovisuales para la traducción, en el caso concreto un lenguaje de la calle con una considerable cantidad de lunfardismos utilizado por los protagonistas de la serie televisiva Okupas. Guiado por la función prioritaria que cumplen las expresiones de lunfardo en dicha serie, se ha detectado el estilo de habla más adecuado en la versión alemana, a saber, un estilo que se basa en los registros juveniles de la lengua meta. A partir de un diálogo elegido se han presentado algunas propuestas de traducción elaboradas en clase con estudiantes de traducción, con el fin de mostrar la viabilidad de soluciones más originales y creativas que van más allá de la práctica usual de neutralizar los rasgos diasistemáticos, transfiriéndolos a la variedad estándar del alemán.

Debido a diferentes factores (presión de tiempo, escasez de recursos económicos, sincronicidad de los labios, expectativas de los espectadores, etc.) en la práctica profesional, el margen para buscar, sopesar y elegir opciones que tomen en cuenta la variación lingüística en el texto de partida suele ser muy reducido. Sin embargo, abogo por experimentar -en la medida de lo posible- con soluciones creativas que sean capaces de transferir al menos parte de los componentes emocionales y ambientales que transporta el uso de la variedad no-estándar en la versión original de la película.

\footnotetext{
${ }^{15}$ Conde también indica "'hortelano', apocopado finalmente en orto y con eliminación de la $h$ inicial [?]” como posible fuente en su diccionario etimológico (2004: 238), aunque esta explicación resulta insatisfactoria en el aspecto semántico.
} 


\section{Bibliografía}

Androutsopoulos, Jannis (2006): „Jugendsprachen als kommunikative soziale Stile. Schnittstellen zwischen Mannheimer Soziostilistik und Jugendsprachforschung“. Deutsche Sprache 6/1-2: 106-121. http://jannisandroutsopoulos.files.wordpress.com/2009/12/fs-kallmey er-final-ds-34-2006-106-121.pdf [22.04.2012].

Bielinsky, Fabián (dir.) (2000): Nueve Reinas. Argentina: Patagonik Film Group/Industrias Audiovisuales Argentinas.

Boon, Danny (dir.) (2008): Bienvenue chez les Ch'tis. France: Pathé Renn Productions/Hirsch/Les Productions du Chicon.

Conde, Oscar (2004): Diccionario etimológico del lunfardo. $2^{\circ}$ edición. Buenos Aires: Taurus.

Corominas, Joan (1954): Diccionario crítico etimológico de la lengua castellana. Vol. I (AC). Bern: Francke.

Cortázar, Julio (1956): Final del juego. Ciudad de México: Los Presentes.

Gobello, José (1990): Nuevo Diccionario de lunfardo. Buenos Aires: Corregidor.

Gobello, José (2004): "Prelunfardismos, paralunfardismos, postlunfardismos" (Tomado del Libro de los treinta años de la Academia Porteña del Lunfardo. Buenos Aires: Fraterna). http://geocities.ws/lunfa2000/ppp.htm [23.10.2017].

Gottero, Laura (2006): Diccionario de lunfardo. Buenos Aires: Andrómeda.

Herbst, Thomas (1994): Linguistische Aspekte der Synchronisation von Fernsehserien. Phonetik, Textlinguistik, Übersetzungstheorie. Tübingen: Niemeyer.

Isola, Laura (s. a.): "Tomar o no tomar". Página112. www.autores.org.ar/efeldman/okupas2 /RADA.htm [14.07.13].

Koch, Peter/Österreicher, Wulf (2011): Gesprochene Sprache in der Romania: Französisch, Italienisch, Spanisch. 2. Auflage. Berlin etc.: de Gruyter.

Mantarro, Chiara (2010): "Traducir el cine, traducir el dialecto: Estudio lingüístico de la película Romanzo Criminale". Entreculturas 2. www.entreculturas.uma.es/n2pdf/artic ulo10.pdf [10.04.2012].

Martín Zorraquino, María Antonia/Portolés Lázaro, José (1999): "Los marcadores del discurso”. En: Bosque, Ignacio/Demonte, Violeta (eds.): Gramática descriptiva del español. Vol. 3. Madrid, Espasa-Calpe: 4051-4213.

Nord, Christiane (2007): Textanalyse und Übersetzen. Theoretische Grundlagen, Methode und didaktische Anwendung einer übersetzungsrelevanten Textanalyse. 3. Auflage. Tübingen: Groos.

Pascual Asensi, Jorge (2007): "El vocativo árabe يا ي ya como posible étimo de la interjección che/xe del castellano y el catalán valencianos: una apostilla a los diccionarios etimológicos". Revista de Filología Románica 24: 153-169.

Pérsico, Eduardo (2004): Lunfardo en el tango y la poética popular. Buenos Aires: Proyecto.

Plass, Kerstin (2013): Nueve Reinas auf Wienerisch. Möglichkeiten und Grenzen der Übersetzung des Lunfardo. Saarbrücken: AV Akademikerverlag.

Pöllmann, Magdalena (2009): „Sprachvarietäten als Herausforderung beim Filmübersetzen”. En: Zybatow, Lew (ed.): Translation: Neue Entwicklungen in Theorie und Praxis. SummerTrans-Lektionen zur Translationswissenschaft. IATI-Beiträge I. Frankfurt a. M. etc., Lang: 212-219. 
Latinpedia. www.latinpedia.net/Hogar/television/Series-de-television-Okupas-ad507.htm [14.07.17].

Rabadán Álvarez, Rosa (1991): Equivalencia y traducción. Problemática de la equivalencia translémica inglés-español. Zamora: Universidad de León.

Stagnaro, Bruno (dir.) (2000): Okupas. Argentina: Ideas del sur.

Teruggi, Mario (1978): Panorama del lunfardo. Génesis y esencia de las hablas coloquiales urbanas. $2^{\circ}$ edición. Buenos Aires: Editorial Sudamericana.

Vermeer, Hans Josef (1992): „Eine kurze Skizze der scenes- \&-frames-Semantik für Translatoren“. En: Salevsky, Heidemarie (ed.): Wissenschaftliche Grundlagen der Sprachmittlung: Berliner Beiträge zur Übersetzungswissenschaft. Otto Kade zum Gedenken. Frankfurt a. M. etc., Lang: 75-83.

Wiktionary. Glosario del lunfardo. https://es.wiktionary.org/wiki/Apéndice:Glosario_ del_lunfardo [14.07.17].

Zimmermann, Klaus (2004): „Äquivalenzgrade der Übersetzung von jugendsprachlichen Texten und die Möglichkeiten der kontrastiven lexikographischen Erfassung der Jugendsprache (am Beispiel des Deutschen und Portugiesischen)“. En: Endruschat, Annette/Schönberger, Axel (eds.): Übersetzung und Übersetzen aus dem und ins Portugiesische. Frankfurt a. M., Domus Editoria Europea: 23-57. 